Establish "managed-care" unit to enforce the delivery of highquality health services

Restructure reimbursement rates for public providers such that they are reflective of public-health need, not cost

Enforcement of gate-keeping at primary care level through patient financial incentives

Consider phased-in prescription benefit for generic pharmaceuticals through accredited private pharmacies

Conclusion PhilHealth is emerging as a defining actor for healthcare in the country. It's legitimacy among the population has grown over the years, it benefits from strong political support, and its purchasing power has grown to one-third of public health expenditures. The study also confirmed that, with several strategy adjustments, PhilHealth has the potential to cover the "missing middle": 14 million uncovered informal workers and their dependents. Altogether, these strategies (see below) have the potential to reach $60 \%$ of the uncovered population. Improving continuity of payment is fundamental.

\title{
053 EXTENDING UNIVERSAL HEALTH COVERAGE FOR THE INFORMAL SECTOR IN PHILIPPINES
}

Ma. Lourdes Macapanpan. International Labour Organization, Manila, Philippines

10.1136/bmjopen-2015-forum2015abstracts.53

Background Philippines is one the most advanced middle-income countries with regard to its achievement in universal health coverage (UHC). Today, $82 \%$ of its population benefits from PhilHealth coverage and has access to public and private hospitals services. But still around 18 million Filipinos, mainly informal workers, are excluded from the system.

Objectives To explore potential strategies to further expand coverage of the Filipino population, as well as to improve PhilHealth's role in setting health quality standards. The study was conducted to provide broad, strategic-level recommendations to guide future directions.

Methods First, a literature review was conducted to analyze existing data and assessments about the health financing system in the Philippines. Second, a one week field visit was undertaken to interview existing and potential partners of PhilHealth. Intensive meetings with a range of departments within PhilHealth were also undertaken. Finally, a validation meeting was held with PhilHealth senior management and Informal Member Management Group to review findings and recommendations.

Result Based on our findings, the team recommended several strategic directions, including:

1. Rationalize iGroup program to maximize enrollment

Harmonize all benefits and policies across iGroup \& IPP

Selectively work with large distributers, instead of smaller groups and simplify incentives

2. Develop comprehensive plan to improve premium continuity

Develop coordinated communication plan with distributers for members transitioning out of their group

Assess possible incentives for renewal at all levels and develop a comprehensive premium continuity plan

3. Improve outcomes through efficient partnerships

Develop partnership management processes and optimal repartition of roles and responsibilities of all actors

Build dedicated vendor support capabilities within PhilHealth

Consider partnering with private health insurance providers to improve benefit package, increase renewals and stimulate innovations

4. Act and advocate to improve the "product" 